\title{
Microwave Ablation With a Triaxial Antenna: Results in ex vivo Bovine Liver
}

\author{
Christopher L. Brace, Student Member, IEEE, Paul F. Laeseke, Daniel W. van der Weide, Member, IEEE, and \\ Fred T. Lee, Jr.
}

\begin{abstract}
We apply a new triaxial antenna for microwave ablation procedures to an ex vivo bovine liver. The antenna consists of a coaxial monopole inserted through a biopsy needle positioned one quarter-wavelength from the antenna base. The insertion needle creates a triaxial structure, which enhances return loss more than $10 \mathrm{~dB}$, maximizing energy transfer to the tissue while minimizing feed cable heating and invasiveness. Numerical electromagnetic and thermal simulations are used to optimize the antenna design and predict heating patterns. Numerical and ex vivo experimental results show that the lesion size depends strongly on ablation time and average input power, but not on peak power. Pulsing algorithms are also explored. We were able to measure a $3.8-\mathrm{cm}$ lesion using $50 \mathrm{~W}$ for $7 \mathrm{~min}$, which we believe to be the largest lesion reported thus far using a 17-gauge insertion needle.
\end{abstract}

Index Terms-Ablation, electromagnetic heating, microwave (MW) ablation, MW heating, RF.

\section{INTRODUCTION}

$\mathbf{S}$ URGICAL resection remains the gold standard treatment for primary or metastatic liver cancer. However, a majority of patients are not eligible for surgery due to a high number of tumors, difficult location of tumors, or associated comorbidities [1]-[4]. This has led to the development of minimally invasive alternatives for the treatment of liver tumors, notably focal tumor ablation. The most popular modality, RF ablation, has been plagued by high post-treatment local recurrence rates [5]-[7]. RF ablation has several drawbacks related to its mode of heating. It uses alternating electrical current to create ionic agitation, which produces frictional heat and subsequent tissue necrosis [8]. Ground pads must be placed on the patient to create a conduction path, leading to a risk of ground pad burns due to improper pad placement or high-input powers. With RF, long treatment times may be necessary due to tissue desiccation and charring. Charring increases the RF impedance and decreases the amount of energy that can be deposited into the tissue, resulting in long treatment times. Finally, current RF systems are

Manuscript received April 21, 2004; revised June 23, 2004. This work was supported by the National Institutes of Health under Grant R21 RR018303-01.

C. L. Brace is with the Electrical and Computer Engineering Department, University of Wisconsin-Madison, Madison, WI 53706 USA (e-mail: clbrace@wisc.edu).

P. F. Laeske and F. T. Lee, Jr. are with the Radiology Department, University of Wisconsin-Madison, Madison, WI 53706 USA (e-mail: plaeseke@ wisc.edu; ftlee@wisc.edu).

D. W. van der Weide is with the Electrical and Computer Engineering Department, University of Wisconsin-Madison, Madison, WI 53706 USA (e-mail: danvdw@engr.wisc.edu).

Digital Object Identifier 10.1109/TMTT.2004.839308 limited to the use of only one probe at a time due to electrical interference between probes. This means that all but the smallest tumors must be treated with sequential overlapping ablations.

Microwave (MW) ablation, like RF ablation, uses localized heating to cause tissue necrosis. However, MW systems can produce greater and more rapid heating and can easily support the use of multiple probes. Compared to RF, MWs have a theoretically broader field of power density, with a correspondingly larger zone of active heating [9]. This may allow for more uniform tumor kill both within a targeted zone and in perivascular tissue. Furthermore, MW heating does not rely on a conduction current path, which eliminates ground pad and charring concerns. Lesion size is mainly limited by the available power and treatment time. Despite these theoretical advantages, current MW systems use probes that are larger than desirable for percutaneous use (13-15 gauge), and create relatively small zones of necrosis $(1.6 \mathrm{~cm})$ relative to insertion size [10]. This has led to the need for multiple overlapping ablations or antenna arrays, both of which require additional procedure time and invasiveness.

Most ablation antennas are fed by coaxial lines, which have an unbalanced design that allows return current flow on the outer conductor. These currents restrict impedance matching. If the antenna's input impedance is not matched to the feed line, too much of the applied power is reflected from the antenna and, hence, not deposited in the tissue. A mismatch also causes standing waves that can overheat the coaxial feed, causing superficial burns or cable failure.

Numerous previous antenna designs have been presented in the literature for MW ablation [11]-[18]. Several of these designs are specifically targeted for cardiac applications [11]-[13], while others are intended for hyperthermia applications, where lower powers and longer treatments times are acceptable [11], [14]. The tipped monopole designs of [11] and [12] have good SAR patterns, but relatively large reflection coefficients that may restrict power handling of the feed and deposition by the antenna. It is well known that power-handling ability of a coaxial line is strongly related to cable diameter. The dipoles presented in [14] and [15] and the choked designs presented in [13] and [16]-[18] are characterized by low reflections and good SAR patterns, but require an insertion up to $1.5 \mathrm{~mm}$ larger in diameter than the feed cable. The smallest profile choke requires a 14-gauge $(2.1 \mathrm{~mm})$ introduction [17].

The triaxial antenna presented in this paper is optimized for power distribution with the smallest possible insertion. The impedance match between the feed line and antenna allows the maximum power rating of a given feed to be used. Feed-line 


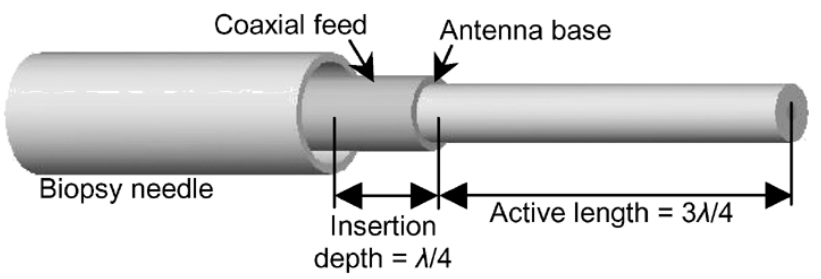

Fig. 1. Schematic representation of a coaxial monopole antenna inserted through a biopsy needle. The active length of the antenna and the insertion depth through the needle may be tuned to account for changes in tissue properties.

TABLE I

Material Properties USED FOR SimUlation AT $37^{\circ} \mathrm{C}, 2.45 \mathrm{GHz}$ [20]

\begin{tabular}{lccccc}
\hline \hline & Liver & $\begin{array}{c}\text { Saline } \\
(0.9 \%)\end{array}$ & PTFE & Copper \\
\hline $\begin{array}{l}\text { Relative } \\
\text { Permittivity }\end{array}$ & $\varepsilon_{r}$ & 45.6 & 78.0 & 1.8 & 1.0 \\
\hline $\begin{array}{l}\text { Electrical } \\
\text { conductivity } \\
(\mathrm{S} / \mathrm{m})\end{array}$ & $\sigma$ & 1.97 & 1.8 & $1.6 \cdot 10^{-5}$ & $5.8 \cdot 10^{7}$ \\
\hline $\begin{array}{l}\text { Thermal } \\
\text { conductivity } \\
(\mathrm{W} / \mathrm{m} \mathrm{K})\end{array}$ & $k_{T}$ & 0.564 & 0.7 & 0.24 & 385 \\
$\begin{array}{l}\text { Specific heat } \\
(\mathrm{J} / \mathrm{kg} \mathrm{K})\end{array}$ & $c_{p}$ & 3400 & 4183 & 1050 & 386 \\
\hline $\begin{array}{l}\text { Density } \\
\left(\mathrm{kg} / \mathrm{m}^{3}\right)\end{array}$ & $\rho$ & 1050 & 998 & 1200 & 9000 \\
\hline \hline
\end{tabular}

heating is minimized. By using the full power rating, more energy may be deposited into the tissue and larger lesions realized. In addition, since the introducing needle is integral to the design, the total insertion diameter remains small enough for safe percutaneous use.

\section{ANTENNA Design AND Numerical Modeling}

\section{A. Antenna Design}

The triaxial antenna design is created from a coaxial monopole and needle together (Fig. 1) [19]. The active length of the antenna loaded in tissue (see Table I for material properties) is nominally $(2 n-1) \lambda / 4$, where $n$ is an integer. The needle sheath creates a triaxial structure and is positioned $(2 n-1) \lambda / 4$ away from the antenna base. This is the insertion depth. Positioning the needle in this way improves return loss and reduces fields flowing back on the coaxial outer conductor. In turn, more energy is deposited in the tissue and less heating of the feed line occurs.

In order to introduce the antenna percutaneously, a biopsy needle with a removable introducer (Bard Medical, Covington, $\mathrm{GA}$ ) is inserted into the patient. Once the proper needle position has been achieved, the introducer is removed. The coaxial antenna is then inserted through the needle and positioned correctly to provide the required tissue heating.

The coaxial feed lines and antennas used with 18- and 17-gauge insertion needles are created from UT-34-M $(0.95 \mathrm{~mm})$ and UT-47-M (1.26 mm) semirigid cable (Micro-Coax, Pottstown, PA), respectively. The entire outer conductor is copper, while the inner conductor is made from silver-plated copper wire. The coaxial dielectric used is a
TABLE II

TEMPERATURE COEFFICIENTS USED IN SIMULATIONS. UNITS ARE $\%{ }^{\circ} \mathrm{C}^{-1}$ UNLESS NOTED OTHERWISE [20], [21]

\begin{tabular}{cccccc}
\hline \hline & $\varepsilon_{r}$ & $\sigma$ & $k_{T}$ & $c_{p}$ & $\rho$ \\
\hline Liver & -0.2 & +1.2 & 0.2 & -0.01 & $0.4 \cdot 10^{-4 \dagger}$ \\
\hline PTFE & -0.003 & +0.01 & 0 & 0 & 0 \\
\hline \hline
\end{tabular}

$\uparrow$ Estimate based on bovine and canine values for muscle.

low-loss polytetrafluoroethylene (PTFE), which results in a still-air power rating of $34 \mathrm{~W}$ for the $0.95-\mathrm{mm}$ cable and $62 \mathrm{~W}$ for the $1.26-\mathrm{mm}$ cable.

The biopsy needle material is stainless-steel coated with $\sim 10 \mu \mathrm{m}$ of Parylene " $\mathrm{C}$ " to reduce the coefficient of friction on the needle surface. Parylene "C" was used because of its biocompatibility, conformal coating, and ease of application.

\section{B. Numerical Modeling}

Heating effects from the antenna are modeled using the finiteelement method (FEM) with multiphysics software (COMSOL Inc., Burlington, MA). The coupled system to be solved is given by the time-harmonic wave equation

$$
\nabla^{2} \boldsymbol{E}=\omega^{2}\left(\varepsilon_{r}(T)-j \frac{\sigma(T)}{\omega}\right) \boldsymbol{E}
$$

and heat-transfer equation

$$
\rho c_{p} \frac{\partial T}{\partial t}=k_{T} \nabla^{2} T+\frac{\sigma}{2}|\boldsymbol{E}|^{2}-Q_{h}
$$

where $\omega$ is the angular frequency (in radians per second), $\boldsymbol{E}$ is the electric field vector (volts per meter), $T$ is local temperature (kelvin), and $Q_{h}$ represents the heat lost to perfusion or gained by metabolic processes. In ex vivo samples, $Q_{h}$ can be ignored since no perfusion or metabolism exists. Material parameters are assumed to be isotropic and homogeneous. Temperature variances of PTFE and liver are included in the model, as outlined in Table II. Temperature dependencies of metallic components and mechanical changes in PTFE are neglected since the resulting effects are relatively small. Since the antenna is axisymmetric, the model is reduced to a two-dimensional space to limit computational burdens.

The simulation is run for application times up to $15 \mathrm{~min}$ with average input powers from 10 to $80 \mathrm{~W}$. Pulsed algorithms using peak powers up to $300 \mathrm{~W}$ and pulse times from $0.1 \mathrm{~ms}$ to $120 \mathrm{~s}$ are also simulated for comparison to ex vivo measurements. Care must be taken when comparing numerical and experimental data since the computer simulation calculates lesion size based on the $60^{\circ} \mathrm{C}$ contour, where cell death occurs within seconds, while post-ablation measurements rely on visual inspection of tissue necrosis. Visual inspection has been shown to be in good agreement with histology [18].

\section{EXPERIMENTAL METHODS}

After validation of the antenna design, several ablation protocols are tested in order to map out the lesion size dependence on independent variables (IVs) such as application time, average power, peak power, and pulse on/off times (Table III). 
TABLE III

Ablation Protocols to BE TESTED ex vivo. “@” INDICATES THE TESTING INTERVAL

\begin{tabular}{|c|c|c|c|c|}
\hline Protocol IV & $\begin{array}{c}\text { Application } \\
\text { time }\end{array}$ & Average power & Peak power & Pulse times \\
\hline $\begin{array}{l}\text { Application } \\
\text { time }\end{array}$ & $\begin{array}{l}1-12 \text { min } \\
\text { a } 1 \text { min }\end{array}$ & $\begin{array}{l}25 \mathrm{~W}(18 \mathrm{ga}) \\
50 \mathrm{~W}(17 \mathrm{ga})\end{array}$ & $N / A$ & $N / A$ \\
\hline $\begin{array}{l}\text { Average } \\
\text { power }\end{array}$ & $10 \mathrm{~min}$ & $\begin{array}{c}5-30 W @ 5 W \\
(18 \mathrm{ga}) \\
5-65 \mathrm{~W} @ 10 \mathrm{~W} \\
(17 \mathrm{ga})\end{array}$ & $N / A$ & $N / A$ \\
\hline Peak power & $8 \mathrm{~min}$ & $40 \mathrm{~W}$ & $\begin{array}{c}40-300 W @ \\
20 \mathrm{~W} \\
\end{array}$ & $\begin{array}{c}0.1 \mathrm{~ms}- \\
120 \mathrm{~s}\end{array}$ \\
\hline Pulse times & $8 \mathrm{~min}$ & $25-40 \mathrm{~W}$ & $\begin{array}{c}40-300 \mathrm{~W} @ \\
20 \mathrm{~W}\end{array}$ & $\begin{array}{c}0.1 m s- \\
120 s\end{array}$ \\
\hline
\end{tabular}

During the testing of each protocol, only the IV is altered, while all other variables are kept as fixed as possible. The forward and reflected powers are continuously measured from the source with $\pm 0.3 \mathrm{~W}$ accuracy.

Finally, a repeatability study is conducted using seven samples and the following parameters.

- $P_{\text {in }}=50 \mathrm{~W}$.

- $t=7 \mathrm{~min}$.

- $T_{0}=37{ }^{\circ} \mathrm{C}$

This study is used to evaluate the variability in lesion size due to any spatial and temporal variations in tissue and cable properties. By keeping the initial temperature constant, we remove the large variance in lesion size caused by changing initial temperature. The results are used in conjunction with thermal modeling to predict in vivo lesion sizes.

At the end of each ablation, tissue temperature is measured from a thermocouple probe inserted into the middle of the lesion to monitor maximum lesion temperature. The lesion is then divided longitudinally along the insertion track. Measurements are taken of the lesion diameter using a straight edge ruler or caliper and are based on visual inspection of the area of necrosis. Measurement accuracy is estimated at $\pm 0.5 \mathrm{~mm}$.

\section{RESULTS}

Both numerical and experimental results show that, for this triaxial design, $\Gamma$ is minimized for a probe length of $12.3 \mathrm{~mm}$ and insertion depth of $3.5 \mathrm{~mm}$ in liver tissue (Fig. 2) and that the distance between the outer conductor and insertion needle is not a critical factor for antenna performance. In fact, having the needle in contact or near contact with the outer conductor provided lower reflection coefficients in both simulated and empirical data [19].

For the coaxial monopole in saline, $\Gamma$ was measured to be $-35.0 \mathrm{~dB}$ at $2.45 \mathrm{GHz}$. When inserted $3.5 \mathrm{~mm}$ through a biopsy needle, $\Gamma$ was reduced to $-46.2 \mathrm{~dB}$. Thus, the triaxial design improved the reflection coefficient by $11.2 \mathrm{~dB}$ over the simple coaxial monopole, as seen in Fig. 3.

$\Gamma$ was then measured to be $-25.0 \mathrm{~dB}(0.32 \%$ reflected power $)$ for an active length of $12.3 \mathrm{~mm}$ and insertion depth of $3.5 \mathrm{~mm}$ in an ex vivo bovine liver. Reflection coefficient is most likely

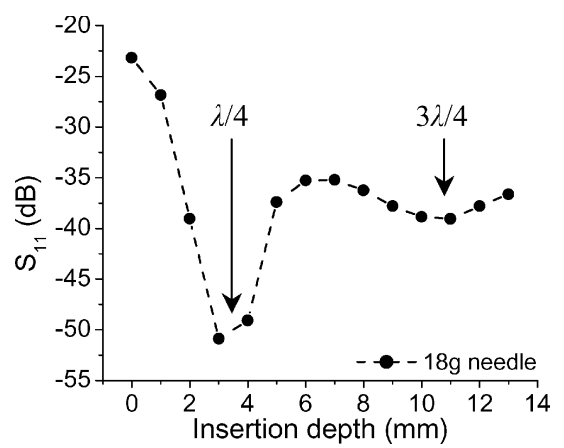

Fig. 2. Insertion depth versus $\Gamma$ measurements. $\Gamma$ is minimized for a $3.5-\mathrm{mm}$ insertion depth in liver tissue [19].

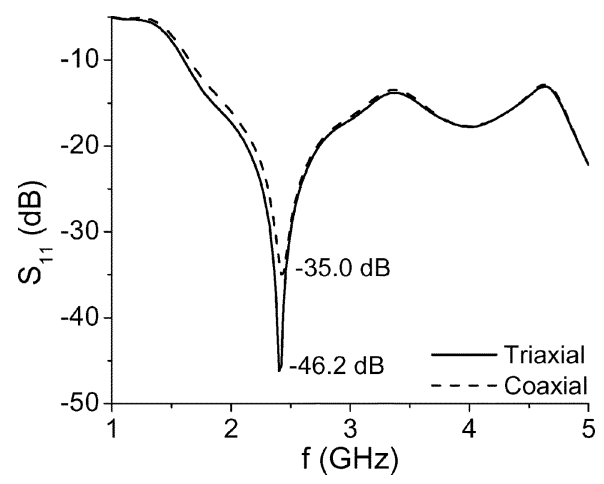

Fig. 3. Measured $S_{11}$ of a coaxial monopole and a triaxial antenna. The triaxial design reduces reflections by $11.2 \mathrm{~dB}$ [19].

limited by inhomogeneity in the dielectric constant of the liver tissue.

\section{A. Lesion-Size Dependence on Application Time}

Experimental data for this study were taken on two successive days. On the first day, initial tissue temperatures were measured to be $18.0^{\circ} \mathrm{C} \pm 0.5^{\circ} \mathrm{C}$, while on the second day initial temperatures were $11.0^{\circ} \mathrm{C} \pm 0.5^{\circ} \mathrm{C}$. Shortly after ablation, temperatures in the zone of necrosis were measured to be in excess of $80^{\circ} \mathrm{C}$, far surpassing the commonly used metric of $\sim 60^{\circ} \mathrm{C}$ for instantaneous cell death. Numerical simulations were carried out with the same initial parameters as in the experiments.

Fig. 4 shows the results of the application time protocol. Lesion diameters increased with time with diminishing increases as application time increased according to the model

$$
Y=Y_{0}+Y_{1} t+Y_{2} \sqrt{t}
$$

where $Y$ is the lesion diameter and $t$ is the application time, a trend that is also noted in simulations. The largest lesion measured was $3.9 \mathrm{~cm}$ in diameter and was limited in size by the initial tissue temperature.

Discrepancies between numerical and experimental values are most likely due to the metrics used and an insufficient tissue model. Recall that simulated lesion size is defined from the $60{ }^{\circ} \mathrm{C}$ contour line, which corresponds indirectly to actual lesion size. More importantly, data from Table II are only characterized in the literature for tissue temperatures up to $\sim 70{ }^{\circ} \mathrm{C}$. Better characterization of tissue properties above $100^{\circ} \mathrm{C}$, where dehydration and charring are likely to reduce heat conduction 


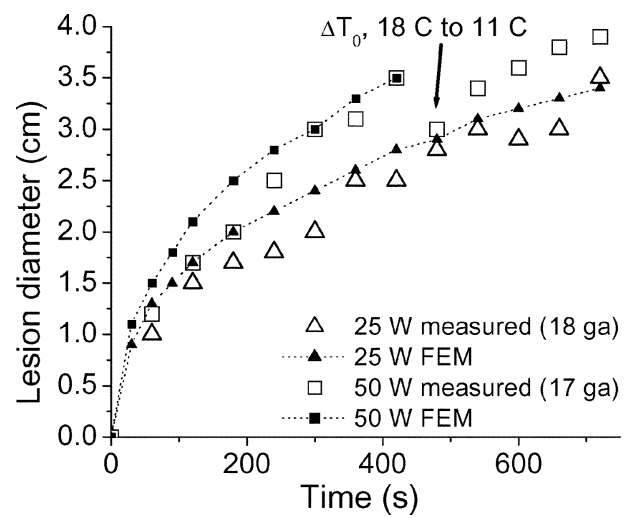

Fig. 4. Ex vivo measurements of lesion diameter versus application time. Note the change of $T_{0}$ in the 18-gauge data set. Lesion size in numerical simulations was defined as the maximum diameter of the $60{ }^{\circ} \mathrm{C}$ contour.

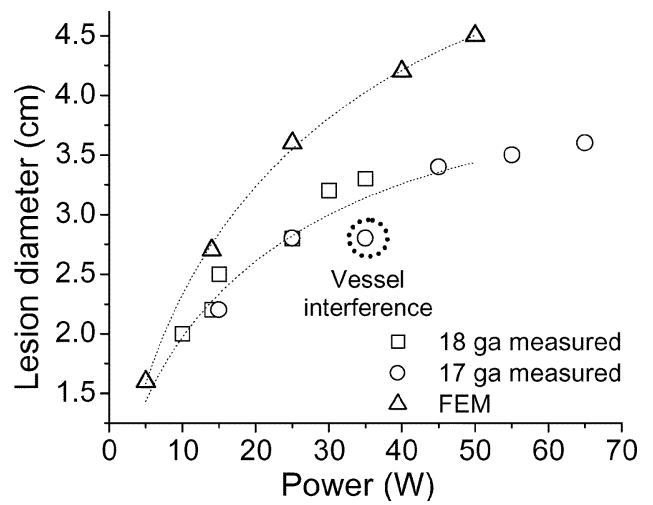

Fig. 5. Ex vivo measurements of lesion diameter at 10 min versus average input power. Simulations were identical for both antenna sizes. The circled point was limited in size due to a large vessel in the active region of the antenna. Dotted lines represent fits of the data to (3).

and bulk electrical conductivity in the tissue, may produce more congruent data between simulation and experiment.

Numerical data also predicts that the same trend will occur for in vivo studies, but that the slope change will decrease faster with time due to the larger effective thermal conductivity, i.e., graphs like Fig. 4 will appear flatter at longer time points, where thermal conduction most directly controls lesion size.

\section{B. Lesion-Size Dependence on Average Input Power}

For this protocol, initial ablation temperatures ranged from $15.0^{\circ} \mathrm{C} \pm 0.5^{\circ} \mathrm{C}$ to $18.0^{\circ} \mathrm{C} \pm 0.5^{\circ} \mathrm{C}$. Again, final temperatures in the core of the lesion were measured to be in excess of $80^{\circ} \mathrm{C}$.

Fig. 5 demonstrates the dependence of lesion size on average input power. We find the same functional dependence as (3) for lesion size and input power in both simulation and experimental results. Again, simulations tended to overestimate the size of the lesions as power increased. The 17-gauge outlier at $35 \mathrm{~W}$ was likely due to a vessel running through the active region of the antenna, as in Fig. 6.

Vessels in ex vivo samples are filled with fluid and air, which create material inhomogeneities, increase reflected power, and reduce the energy deposited in the tissue. This likely caused the smaller lesion size. Many of the data points in these protocols had very inhomogeneous active regions-mostly due to empty vessels and bile ducts-and were not the ideal cases studied in

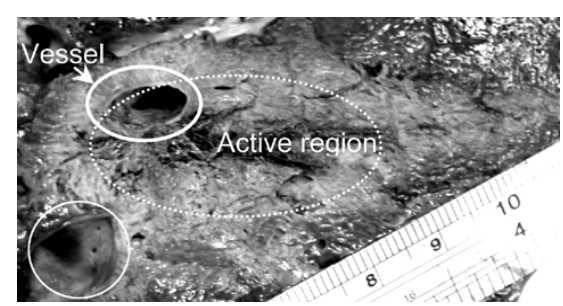

Fig. 6. Example of vessels running through the active region of the antenna. Vessels cause inhomogeneity and anisotropy of the antenna medium and, thus, alter the radiation characteristics, reduce the impedance match, and reduce power deposition into the tissue.

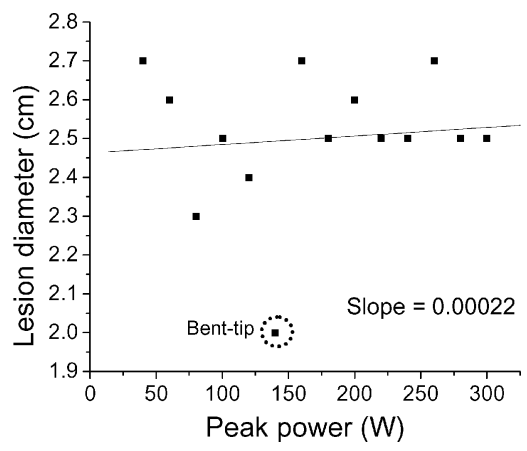

Fig. 7. Ex vivo measurements of lesion diameter versus peak power with average power held constant at $25 \mathrm{~W}$. The slope of the best linear fit is nearly zero. Thermal simulations give similar results-that the lesion diameter depends primarily on the energy applied, not the power.

computer simulations. The effects of inhomogeneities on the resulting lesions can be seen, but since no liver will present a perfectly homogeneous medium, all of the data points measured are included here.

\section{Lesion-Size Changes With Pulsed Power and Pulse Time}

The final protocols provided very little change from previous results. There seems to be no dependence of lesion diameter on peak applied power (Fig. 7). Similar amounts of energy were deposited into the active region of the antenna because of the fixed average power and application time. This makes qualitative sense since total energy, not power, is the appropriate metric for measuring heat generation. Thus, in this case, we have a large sample of $25-\mathrm{W}$ lesions created in tissues with similar initial temperatures of $16{ }^{\circ} \mathrm{C}-18{ }^{\circ} \mathrm{C}$. The mean diameter in this case is $2.5 \mathrm{~cm}$ and the standard deviation is 0.184 . One data point is considered an outlier because the antenna was unknowingly bent during insertion. If we ignore the bent-tip data point at $140 \mathrm{~W}$, the average increases slightly to $2.54 \mathrm{~cm}$, while the standard deviation improves to $0.119 \mathrm{~cm}$.

Changing the pulse times did very little to improve lesion diameter, which depended mostly on the average power applied. When pulses from 15 to $120 \mathrm{~s}$ were used with duty cycles above 50\%, lesions decreased somewhat in size as duty cycle decreased. The most notable feature of this experiment was that lesion diameter decreased more rapidly when duty cycle used was less than $50 \%$. These trends were also observed in the thermal simulations. The largest lesions were created with large duty cycles, indicating that simply using constant power is a better option. 


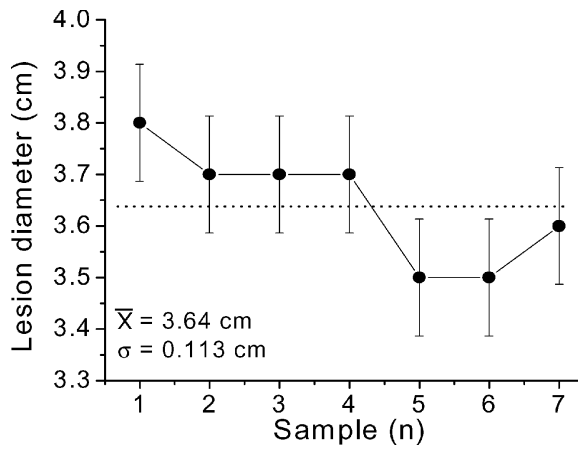

Fig. 8. Repeatability of lesion size. Lesions were created with $50 \mathrm{~W}$, continuous, for $7 \mathrm{~min}$ using the triaxial antenna. The dashed line at $3.64 \mathrm{~cm}$ represents the mean value.

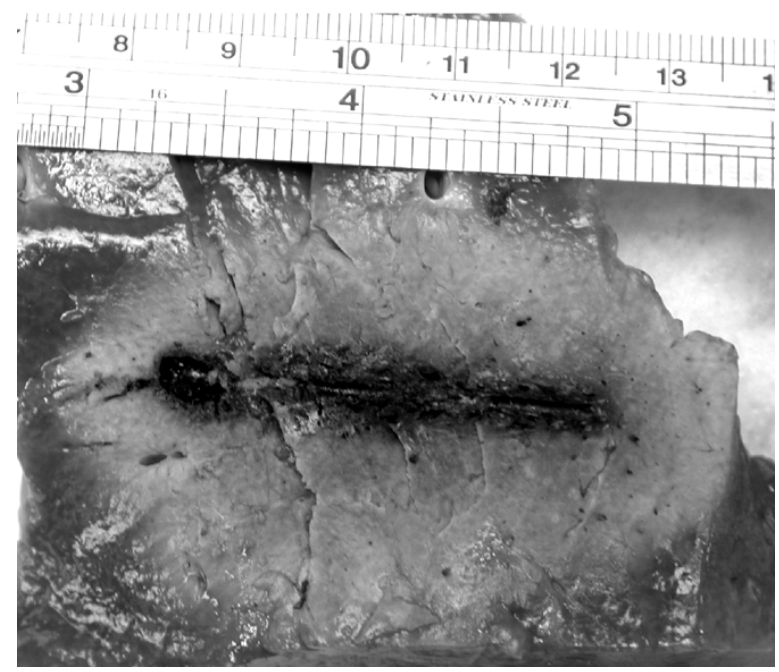

Fig. 9. 3.8-cm lesion created with $50 \mathrm{~W}$ for $7 \mathrm{~min}$.

The effect of this type of pulsing in vivo has yet to be studied, but because the cooling times are assumed to be faster in vivo, some sort of pulsing algorithm may be warranted. For example, a relatively short high-power pulse might be applied to coagulate a blood vessel and reduce the effects of perfusion. Lower $\mathrm{CW}$ power might then be used to create the appropriate zone of necrosis. Using pulsed powers may also reduce fatigue in the feeding cables and antennas, which may result in a more robust system.

\section{Repeatability Study}

Results of the repeatability study may be found in Fig. 8. In this study, the mean lesion diameter $(\bar{x})$ was found to be $3.64 \mathrm{~cm}$ with a standard deviation $(\sigma)$ of $0.113 \mathrm{~cm}$, similar to the results obtained in the peak-power study. We also note that the lesion size tends to decrease slightly with each successive ablation and attribute this fact, in part, to temporal changes in the feed cable induced by temperature variations. An image of the largest lesion from this study is shown in Fig. 9 for reference.

\section{CONCLUSION}

A triaxial antenna design for clinical MW ablation was tested in an ex vivo bovine liver. Previous numerical and experimental data provided show that the triaxial antenna achieves return losses as low as $-46.2 \mathrm{~dB}$ in saline and $-25.0 \mathrm{~dB}$ in an ex vivo bovine liver tissue, with the triaxial design improving return losses by up to $11.2 \mathrm{~dB}$ over a coaxial design. This matching allows high powers to be used in relatively small diameter antennas for procedures at least $12 \mathrm{~min}$ in length. The antenna is capable of being presented inside 18- or 17-gauge needles, making percutaneous introduction feasible.

Ex vivo data compiled show that lesion size is a function of both average input power and application time. Since average input power is limited by the power-handling capability of the coaxial feed line, improvement is possible with larger cables, cables with lower loss, or better heat transfer characteristics. However, larger cables are not desirable due to their invasive nature.

No significant dependence was observed on peak power. Using pulse on/off times $\sim 10 \mathrm{~s}$ was not beneficial in ex vivo simulations or measurements and, in fact, decreased lesion size when the duty cycle fell below $50 \%$.

We found that an average lesion diameter of $3.64 \mathrm{~cm}$ is possible using a conservative $50 \mathrm{~W}$ of input power for $7 \mathrm{~min}$, which makes MW ablation a viable surgical alternative. Thermal modeling predicts that the lesion size will decrease $\sim 25 \%$ for this time-power protocol in vivo. If $65 \mathrm{~W}$ is able to be used in vivo, then lesion diameters near $\sim 3.0 \mathrm{~cm}$ are predicted. Further testing in vivo is warranted and ongoing.

Comparisons between datasets must be taken with care. Both simulated and measured data suggest that changing the initial tissue temperature from $\sim 10{ }^{\circ} \mathrm{C}$ to $\sim 25{ }^{\circ} \mathrm{C}$ results in a $\sim 30 \%$ change in lesion size, clearly demonstrating the influence of initial conditions.

\section{ACKNOWLEDGMENT}

The authors wish to thank D. Haemmerich, Medical University of South Carolina, Charleston, for discussions related to this work.

\section{REFERENCES}

[1] B. Gerard and H. Bleiberg, "Treatment of hepatocarcinoma," Curr. Oncol. Rep., vol. 6, no. 3, pp. 184-191, May 2004.

[2] J. M. Llovet, A. Burroughs, and J. Bruix, "Hepatocellular carcinoma," Lancet, vol. 362, pp. 1907-1917, Dec. 2003.

[3] S. Evrard, Y. Becouarn, M. Fonck, R. Brunet, S. Mathoulin-Pelissier, and V. Picot, "Surgical treatment of liver metastases by radiofrequency ablation, resection, or in combination," Eur. J. Surg. Oncol., vol. 30, no. 4, pp. 399-406, May 2004.

[4] S. A. Curley, F. Izzo, E. Abdalla, and J. N. Vauthey, "Surgical treatment of colorectal cancer metastasis," Cancer Metastasis Rev., vol. 23, no. 1-2, pp. 165-182, Jan.-Jun. 2004.

[5] Y. Komorizono, M. Oketani, K. Sako, N. Yamasaki, T. Shibatou, M. Maeda, K. Kohara, S. Shigenobu, K. Ishibashi, and T. Arima, "Risk factors for local recurrence of small hepatocellular carcinoma tumors after a single session, single application of percutaneous radiofrequency ablation," Cancer, vol. 97, no. 5, pp. 1253-1262, Mar. 2003.

[6] A. Siperstein, A. Garland, K. Engle, S. Rogers, E. Berber, A. Foroutani, A. String, T. Ryan, and P. Ituarte, "Local recurrence after laparoscopic radiofrequency thermal ablation of hepatic tumors," Ann. Surg. Oncol., vol. 7, no. 2, pp. 106-113, Mar. 2000.

[7] L. Solbiati, T. Livraghi, S. N. Goldberg, T. Ierace, F. Meloni, M. Dellanoce, L. Cova, E. F. Halpern, and G. S. Gazelle, "Percutaneous radiofrequency ablation of hepatic metastases from colorectal cancer: Longterm results in 117 patients," Radiology, vol. 21, no. 1, pp. 159-166, Oct. 2001. 
[8] S. N. Goldberg, G. S. Gazelle, and P. R. Mueller, "Thermal ablation therapy for focal malignancies: A unified approach to underlying principles, techniques, and diagnostic imaging guidance," Amer. J. Roentgenol., vol. 174, pp. 323-331, 2000.

[9] J. C. Lin, Y.-L. Wang, and R. J. Hariman, "Comparison of power deposition patterns produced by microwave and radio frequency cardiac ablation catheters," Electron. Lett., vol. 30, no. 12, pp. 922-923, 1994.

[10] T. Seki et al., "Ultrasonically guided percutaneous microwave coagulation therapy for small hepatocellular carcinoma," Cancer, vol. 74, pp. 817-825, 1994.

[11] M. F. Iskander and A. M. Tumeh, "Design optimization of interstitial antennas," IEEE Trans. Biomed. Eng., vol. 36, no. 2, pp. 238-46, Feb. 1989.

[12] S. Labonte, H. Ali, and L. Roy, "Monopoles for microwave catheter ablation of heart tissue," in IEEE MTT—S Int. Microwave Symp. Dig., 1995, pp. 303-306.

[13] H.-M. Chiu, A. S. Mohan, A. R. Weily, D. J. R. Guy, and D. L. Ross, "Analysis of a novel expanded tip wire (ETW) antenna for microwave ablation of cardiac arrhythmias," IEEE Trans. Biomed. Eng., vol. 50, no. 7, pp. 890-899, Jul. 2003.

[14] W. Hurter, F. Reinbold, and W. J. Lorenz, "A dipole antenna for interstitial microwave hyperthermia," IEEE Trans. Microw. Theory Tech., vol. 39, no. 6, pp. 1048-1054, Jun. 1991.

[15] K. Saito, S. Hosaka, S.-Y. Okabe, H. Yoshimura, and K. Ito, “A proposition on improvement of a heating pattern of an antenna for microwave coagulation therapy: Introduction of a coaxial-dipole antenna," Elec. Commun. Jpn., vol. 86, no. 1, pp. 16-23, 2003.

[16] J. C. Lin and Y.-J. Wang, "The cap-choke catheter antenna for microwave ablation treatment," IEEE Trans. Biomed. Eng., vol. 43, no. 6 , pp. 657-660, Jun. 1996.

[17] I. Longo, G. B. Gentili, M. Cerretelli, and N. Tosoratti, "A coaxial antenna with miniaturized choke for minimally invasive interstitial heating," IEEE Trans. Biomed. Eng., vol. 50, no. 1, pp. 82-88, Jan. 2003.

[18] S. A. Shock, K. Meredith, T. F. Warner, L. A. Sampson, A. S. Wright, T. C. Winter, III, D. M. Mahvi, J. P. Fine, and F. R. Lee, Jr., "Microwave ablation with loop antenna: In vivo porcine liver model," Radiology, vol. 231, no. 1, pp. 143-149, Apr. 2004.

[19] C. L. Brace, D. W. van der Weide, F. T. Lee, P. F. Laeseke, and L. Sampson, "Analysis and experimental validation of a triaxial antenna for microwave tumor ablation," in IEEE MTT-S Int. Microwave Symp. Dig., 2004, pp. 1437-1440.

[20] F. A. Duck, Physical Properties of Tissue: A Comprehensive Reference Book. San Diego, CA: Academic, 1990.

[21] J. Krupka, K. Derzakowski, B. Riddle, and J. Baker-Jarvis, "A dielectric resonator for measurements of complex permittivity of low loss dielectric materials as a function of temperature," Meas. Sci. Technol., vol. 9, pp. 1751-1756, 1998.

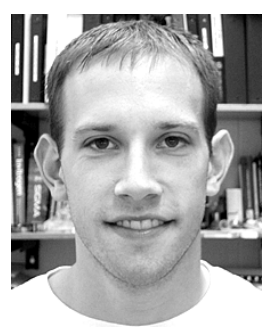

Christopher L. Brace (S'01) was born in Janesville, WI, in 1978. He received the B.S. degree in physics and B.S.E.E. degree from the University of Wisconsin-Milwaukee, in 2001, the M.S.E.E. degree from the University of Wisconsin-Madison, in 2003, and is currently working toward the Ph.D. degree in electrical engineering from the University of Wisconsin-Madison.

He has held summer positions with G.E. Medical Systems in magnetic resonance, digital X-ray detection, and computed tomography. His main research interests include medical applications of MWs, thermal treatment of tumors, and medical imaging.

Mr. Brace is a student member of the American Physical Society. He was a finalist in the 2004 Student Paper Competition of the IEEE Microwave Theory and Techniques Society (IEEE MTT-S) International Microwave Symposium (IMS).

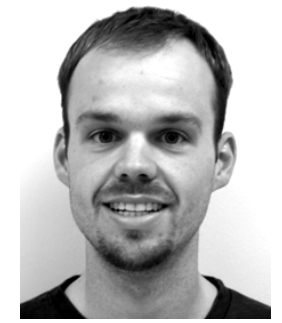

Paul F. Laeseke was born in Madison, WI, on May 31, 1978. He received the B.S. degree in biomedical engineering from the University of Wisconsin-Madison, in 2001, and is currently working toward the M.B.B.S./Ph.D. degree at the University of Sydney, Sydney, Australia.

He has been an Associate Research Specialist, Laboratory Assistant, Biological Science Laboratory Technician, and is currently a Research Intern with the Radiology Department, Medical School, University of Wisconsin-Madison. His current research interests are in multiple probe thermal tumor ablation.

Mr. Laeseke is a member of the American Medical Student Association, the Australian Medical Student Association, and the Australian Medical Association.

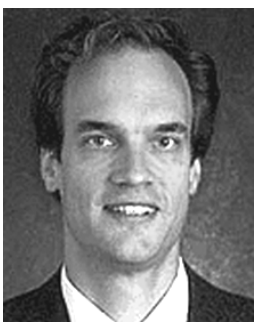

Daniel W. van der Weide (S'86-M'86) received the B.S.E.E. degree from the University of Iowa, Iowa City, in 1987, and the Master's and Ph.D. degrees in electrical engineering from Stanford University, Stanford, CA, in 1989 and 1993, respectively.

He has held summer positions with Lawrence-Livermore National Laboratory and the Hewlett-Packard Company, and full-time positions with Motorola as an Engineer and the Watkins-Johnson Company as a Member of the Technical Staff. From 1993 to 1995 , he was a Post-Doctoral Researcher with the Solid-State Research Department, Max-Planck-Institut für Festkörperforschung, Stuttgart, Germany, after which he joined the University of Delaware, Newark. He is currently a Professor with the Department of Electrical and Computer Engineering, University of Wisconsin-Madison.

Dr. van der Weide was the recipient of the National Science Foundation CAREER and PECASE Awards in 1997 and the Office of Naval Research Young Investigator Program Award in 1998.

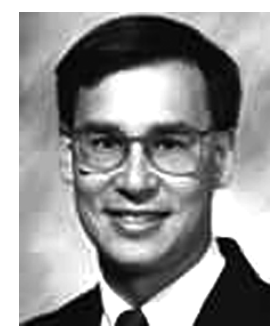

Fred T. Lee, Jr. was born in Okinawa, Japan, on December 31, 1961. He received the B.A. degree in biomedical science and M.D. degree from Boston University, Boston, MA, both in 1986.

He has served as Interim Chief of Ultrasound and was a General Electric/Radiologic Society of North America Scholar. He is currently Chief of Abdominal and Oncologic Imaging and Associate Professor of Radiology with tenure, a member of the Wisconsin Comprehensive Cancer Center and an Affiliate Scientist at the Regional Primate Research Center, University of Wisconsin-Madison. His research interests are in thermal tumor ablation.

Dr. Lee is a member of the American College of Radiology, Radiologic Society of North America, Association of University Radiologists, American Institute of Ultrasound in Medicine, Wisconsin Medical Society, American Roentgen Ray Society, Dane County Medical Society, European Society of Uroradiology, Society of Uroradiology, Society of Urologic Cryosurgeons, Society of Computed Body Tomography/magnetic resonance imaging (MRI), and Society of Gastrointestinal Radiology. 